\title{
L'interférence de l'anglais sur le français chez les apprenants canadiens du français langue seconde
}

\section{Abdulghani Al-Hajebi}

\section{OpenEdition}

12 Journals

Édition électronique

URL : https://journals.openedition.org/rdlc/6788

DOI : $10.4000 /$ rdlc.6788

ISSN : 1958-5772

Éditeur

ACEDLE

Référence électronique

Abdulghani Al-Hajebi, «L'interférence de l'anglais sur le français chez les apprenants canadiens du français langue seconde », Recherches en didactique des langues et des cultures [En ligne], 16-2 | 2019, mis en ligne le 05 septembre 2019, consulté le 21 septembre 2021. URL : http:// journals.openedition.org/rdlc/6788; DOI : https://doi.org/10.4000/rdlc.6788

\section{(c) $(1) \odot$}

Recherches en didactique des langues et des cultures is licensed under a Creative Commons AttributionNonCommercial-NoDerivatives 4.0 International License 


\title{
L'interférence de l'anglais sur le français chez les apprenants canadiens du français langue seconde
}

\author{
Abdulghani Al-Hajebi ${ }^{1}$
}

\begin{abstract}
RÉSUMÉ :
L'apprenant ${ }^{2}$ canadien du français langue seconde possède un ou plusieurs systèmes linguistiques. L'interférence des connaissances de ces systèmes antérieurs, en particulier l'anglais pour notre étude, pose un problème à l'apprentissage du français langue seconde. Ce travail se propose d'identifier les types d'interférences que l'anglais exerce sur le français chez les employés de la fonction Publique du Canada apprenant le FLS et d'exposer les sources de ces erreurs. Nous ne proposerons pas des solutions pour ces interférences, mais nous suggérons des mesures qui permettent de les prévenir et de diminuer les interférences fréquentes.
\end{abstract}

\begin{abstract}
:
The Canadian learner of French as a second language has one or more language systems. The interference of knowledge of these prior systems, especially English for our study, poses a problem for learning French as a second language. This work aims to identify the types of interference that English has on French among employees of the Canada's public service learning FSL and to expose the sources of these errors. We will not propose solutions for these interferences, but we suggest measures that can prevent them and reduce frequent interferences.
\end{abstract}

MOTS-CLÉS : interférence, anglais, français langue seconde, linguistique contrastive, enseignement-apprentissage, andragogie

KEYWORDS: interference, English, French as a second language, contrastive linguistics, teaching-learning, andragogy.

\section{INTRODUCTION}

L'interférence est un phénomène linguistique issu du contact de deux ou plusieurs langues. Lors du processus d'apprentissage de langue seconde, l'apprenant se réfère naturellement aux automatismes acquis dans sa langue première. Et ce processus d'apprentissage de la langue seconde passe, selon Troubetzkoy (1939) dans les Principes de phonologie $e^{3}$, par le « crible phonologique » de sa langue première. Ce processus reste inévitable. Ainsi, surgissent au cours de l'apprentissage d'une langue seconde les phénomènes d'interférences que décrivent des linguistes, comme Weinreich dans son ouvrage Languages in contact (1953). L'interférence exige qu'il y ait au moins deux

\footnotetext{
1 Professeur et chercheur en langue et littérature française, London, Ontario, Canada. abdulghani.alhajebi@yahoo.fr

${ }^{2}$ Le masculin dans cet article est utilisé pour alléger le texte, et ce, sans préjudice à la forme féminine.

${ }^{3}$ Troubetzkoy, N., Principes de phonologie, Paris, Klincksieck, 1939.
} 
langues en contact. D'après Darbelnet $(1976: 77)^{4}$, elle est plus perceptible selon que les langues en contact sont apparentées ou non. D'un point de vue linguistique, l'interférence est définie comme accident de bilinguisme en raison du contact entre les langues. Mackey (1976) définit ce phénomène de façon très simple: "l'interférence est l'utilisation d'éléments appartenant à une langue tandis que l'on en parle ou que l'on en écrit une autre $»^{5}$. Dans l'avis de Stockwell, Bowen et Martin (1965), l'interférence linguistique provoque trop de difficultés pédagogiques sur les plans phonétique, morphologique, sémantique et syntaxique. Mais ces auteurs maintiennent que ces difficultés rencontrées ne peuvent pas rendre impossible l'apprentissage d'une nouvelle langue.

L'apprenant d'une langue seconde commet certainement des erreurs dont certaines proviennent de sa langue première. Il procède souvent, pendant sa production orale ou écrite, par un processus d'un transfert de sa langue première à la langue seconde. Ce processus de transfert est adéquat lorsque les deux structures sont semblables; par exemple, l'ordre des mots, la prononciation, ou le vocabulaire. Dans ce cas, on parle d'un transfert positif. Par contre, si les deux structures sont différentes, il s'agit ici d'un transfert négatif qui donnera lieu à l'interférence. Doca (1981) distingue le transfert positif et l'interférence dans les mots suivants : «le transfert désigne les effets du transfert positif ou de facilitation, tandis que l'interférence désigne le transfert négatif ou d'inhibition ${ }^{6}$. » Par l'interférence, nous entendons donc l'utilisation des éléments d'une langue dans le discours d'autre langue. C'est ce que nous allons démontrer dans cette étude.

C'est lors notre expérience d'enseignement de français langue seconde (FLS) dans quelques écoles de formation linguistique pour adultes à Gatineau (Québec) et Ottawa (Ontario), que nous avons constaté que beaucoup d'erreurs faites par les apprenants du FLS sont des interférences de l'anglais sur le français. De ce constat, nous nous proposons de formuler une étude sur cette question d'interférence chez les fonctionnaires fédéraux, apprenants du français langue seconde. Nous avons fait nos observations informelles sur plusieurs apprenants de plusieurs niveaux pour comprendre les types d'erreurs et leurs origines. Notre méthodologie de recherche se base donc sur l'observation participante dans les classes de cours. C'est une observation authentique, hors de toute situation explicite et formelle d'enquête. Avant de procéder à l'analyse des erreurs interférentielles, il est indispensable de comprendre tout d'abord le contexte dans lequel cette étude a été menée ainsi que le dispositif d'enseignement utilisé.

\section{CONTEXTE DE L'ÉTUDE ET DISPOSITIF DE L'ENSEIGNEMENT}

Dans le but d'esquisser le portrait le plus complet et le plus représentatif possible des interférences de l'anglais sur le français dans une classe de FL2, il est paru nécessaire d'étudier le contexte dans lequel nous avons relevé notre corpus ainsi que l'outil andragogique d'enseignement que nous avons utilisé. Il importe également de démontrer les principes andragogiques qui jouent un rôle essentiel dans ce type d'enseignement aux adultes.

\footnotetext{
${ }^{4}$ Darbelnet, Jean, Le français en contact avec l'anglais en Amérique du Nord, Québec, Presses de l'Université Laval, 1976, p. 77.

${ }^{5}$ Mackey, William. F., Bilinguisme et contact des langues, Edition Klincksieck, Paris 1976, p. 414.

${ }^{6}$ Doca, Gheorphe, Analyse psycholinguistique des erreurs faites lors de l'apprentissage d'une langue étrangère, publication de la Sorbonne, 1981, p. 23.
} 
Il faut d'abord préciser que nos étudiants sont des employés de la fonction publique du Canada. Tous ces employés qui apprennent le français langue seconde, qui nous ont permis, en tant qu'apprenants présents dans nos cours, de faire nos observations, ont l'anglais comme première langue officielle, et non pas forcement comme langue maternelle, car certains d'entre eux ont une langue maternelle autre que l'anglais mais qui n'est pas utilisée au travail. Selon le rapport mondial sur l'apprentissage et l'éducation des adultes (GRALE) de l'UNESCO (2012), la population canadienne indique plus de 200 langues et que ceux qui n'ont ni l'anglais ni le français comme langue maternelle représentent $20 \%$ de la population ${ }^{7}$. Cela veut dire que tous les employés qui apprennent le français langue seconde ont l'anglais comme première langue officielle et qu'un pourcentage considérable des fonctionnaires parle une autre langue maternelle que l'anglais. Mais l'interférence dont il est question ici comprend la manifestation des caractères de l'anglais, à l'oral, à l'écrit et à la compréhension écrite, dans la langue seconde. Ce type d' ' erreurs » est communément appelé dans les salles de cours à Ottawa "l'anglicisme ». L'anglicisme est un mot ou une expression empruntée à l'anglais, dont la fréquence d'utilisation en français est élevée, il est ainsi considéré comme étant intégré au lexique du français et donc être inscrit dans les dictionnaires et glossaires. Selon Bogaards (2008), "le français comprend entre 2000 et 4000 mots et expressions d'origine anglaise ${ }^{8}$. » Or dans notre recherche, nous parlons des erreurs faites par des apprenants anglophones et ces erreurs ne font pas souvent partie des mots et expressions inscrits dans les dictionnaires d'anglicisme. Il ne s'agit pas ici d'anglicisme mais d'interférence. Quant à la nature de l'interférence, nous avons écarté dans notre étude les erreurs propres au système de la langue seconde afin de sélectionner les erreurs proprement interférentielles.

Concernant l'utilisation des langues officielles aux institutions fédérales, la Loi sur les langues officielles au Canada (1988) exige des services au public dans les deux langues officielles. Ainsi les institutions fédérales sont obligées à offrir des services en français aux minorités francophones hors Québec, forçant un certain nombre de fonctionnaires fédéraux à apprendre le français comme langue seconde. L'article 22 de cette loi mentionne que :

Il incombe aux institutions fédérales de veiller à ce que le public puisse communiquer avec leur siège ou leur administration centrale, et en recevoir les services, dans l'une ou l'autre des langues officielles. Cette obligation vaut également pour leurs bureaux - auxquels sont assimilés, pour l'application de la présente partie, tous autres lieux où ces institutions offrent des services - situés soit dans la région de la capitale nationale, soit là où, au Canada comme à l'étranger, l'emploi de cette langue fait l'objet d'une demande importante".

\footnotetext{
${ }^{7}$ Conseil des ministres de l'Éducation (Canada), Apprentissage et éducation des adultes. Rapport d'étape du Canada en vue du Rapport mondial sur l'apprentissage et l'éducation des adultes (GRALE) de l'UNESCO et de la fin de la Décennie des Nations Unies pour l'alphabétisation (DNUA), Toronto, 2012.

${ }^{8}$ Paul Bogaards, On ne parle pas franglais, De boeck et Duculot, Bruxelles, 2008, p. 67.

${ }^{9}$ Canada. Loi sur les langues officielles, L.C. juillet 1988, ch. 38, partie IV, art. 21, dernière modification le 21 septembre 2017, à jour le 6 juin, 2019, Ministère de la Justice, 2017. La Loi est accessible en format PDF au lien suivant : https://laws-lois.justice.gc.ca/PDF/O-3.01.pdf
} 
L'apprentissage d'une langue seconde dans les institutions fédérales n'est pas le choix de l'employé, mais c'est l'exigence du poste qui est désigné comme bilingue. En effet, à la fonction publique du Canada, tous les postes sont identifiés comme étant unilingues ou bilingues. Les postes unilingues sont désignés " français essentiel », « anglais essentiel » ou " français ou anglais essentiel ». Les postes bilingues exigent l'usage du français et de l'anglais. Dans le cas des postes bilingues, il existe trois compétences générales en langue seconde : la compréhension de l'écrit, l'expression écrite et l'interaction orale avec trois niveaux demandés $(\mathrm{A}, \mathrm{B}, \mathrm{C})$ pour chaque compétence. Certains postes exigent le niveau le plus élevé $C / C / C$, d'autres $C / B / C$ ou $\mathrm{B} / \mathrm{B} / \mathrm{B}, \ldots$ etc. Il existe aussi deux autres résultats possibles du test : $\mathrm{X}$ et $\mathrm{E}$. Le premier signifie que la performance de l'employé ne satisfait pas aux exigences minimales du niveau A. Quant au niveau E, il signifie que l'employé est exempté indéfiniment de subir d'autres tests linguistiques dans cette habileté. Pour doter un poste qui exige un niveau linguistique dans la langue seconde, la fonction publique attribue une formation linguistique au candidat de ce poste. L'employé suit donc une formation de la langue seconde très souvent pour passer un examen de niveau. Comme nous l'avons mentionné plus haut, tous nos apprenants parlent l'anglais comme langue première. Parmi eux, il y en a qui parlent le français depuis l'enfance, mais le niveau de restriction dans l'emploi du français dans les bureaux les empêche de maitriser complètement tous les aspects du français. En effet, on conçoit volontiers que de tels apprenants recourent à l'anglais pour y puiser les solutions que cette langue peut apporter aux aspects du français qui leur causent des problèmes. C'est ainsi que les transferts négatifs se produisent.

Ensuite, qu'en est-il du dispositif d'enseignement utilisé pendant nos cours? À ce sujet, il convient de préciser que nous utilisions pour nos cours de français langue seconde un dispositif d'enseignement intitulé Programme de française langue seconde (PFL2). Cet outil andragogique est élaboré par l'École de la fonction publique du Canada pour ses employés dont la première langue officielle est l'anglais et qui doivent accomplir ou pourraient avoir à accomplir, en français, des fonctions ou des tâches professionnelles. Ce programme de formation est basé sur une approche communicative et permet aux apprenants d'acquérir les compétences nécessaires pour interagir au milieu de travail et au quotidien. Il couvre les 3 niveaux demandés aux employés du gouvernement fédéral: A, B et C. Les deux premiers niveaux (A et B) comprend 40 objectifs de formation (OF) qui sont conçus pour que l'apprenant atteigne ou maintienne le niveau de compétence B.

Ces OF sont regroupés en quatre grandes étapes. Les trois premières étapes, qui regroupent les trente-deux premiers $O F$, visent l'atteinte du niveau $A$ et la dernière étape, qui regroupe les huit derniers $O F$, l'atteinte du niveau $B$. Chaque objectif de formation se subdivise en capsules qui constituent la phase Appropriation(École de la fonction publique du Canada, Présentation des composantes, Programme de français langue seconde, Niveau $A$ et $B$, Ministre des Travaux publics et Services gouvernementaux du Canada, 2006, p. 6) ${ }^{10}$.

\footnotetext{
${ }^{10}$ Pour consulter le cahier Présentation des composantes en ligne en format PDF, cliquez sur le lien suivant: http://publications.gc.ca/collections/collection_2014/efpc-csps/SC102-2-49-1-2006-fra.pdf.
} 
L'étape d'appropriation comprend huit capsules: Notions, Grammaire, Fonctions, Phonétique, Lexique, Compréhension de l'oral, Stratégies de communication et Langue écrite. Chaque objectif est représenté dans un cahier et le Guide pédagogique $(2006)^{11}$ de ce programme suggère de consacrer une quinzaine d'heures pour chaque objectif.

Quant au niveau C, il comprend deux sessions préparatoires ( 1 et 2) et 4 modules $^{12}$. Ce matériel du PFL2 - C représente 12 semaines de cours : 240 heures de formation, à raison de quatre heures par jour. Ces sessions et modules sont conçus pour que l'apprenant atteigne ou maintienne le niveau de compétence C.

Les cours du programme PFL2 sont souvent dispensés dans des écoles de langues spécialisées, des universités et collèges qui ont des contrats avec le Gouvernement fédéral. Parfois, certaines institutions fédérales exigent la dispense de cours dans leurs locaux. Ce matériel de formation existe uniquement en format électronique et les institutions d'enseignement qui ont des contrats de formation ont l'accès pour en imprimer et faire des copies. Ce matériel contient des centaines d'activités écrites, des enregistrements sonores et visuels et des activités de préparation à l'évaluations de l'écrit et de l'oral ${ }^{13}$.

Nous avons relevé les interférences par l'observation dans une classe de 7 apprenants à l'École de langues Graybridge Malkam à Gatineau (Québec) et trois courts individuels à l'École Knowledge Circle à Ottawa (Ontario). Le tableau 1 présente le recueil des donnés des apprenants sur lesquels nous avons fait notre observation pour cette étude. Les deux écoles de langues sont très réputées pour ce programme et chacune offre des cours du PFL2 au profit d'une centaine d'employés par mois. Ces employés de la fonction publique du Canada sont des directeurs, gestionnaires, avocats, agents de projets, comptables et analystes. Certains sont à temps plein et d'autres à temps partiel selon l'objectif à atteindre. La durée d'un seul cours est de 3 à 3.5 heures et les apprenants inscrits à temps plein ont deux cours par jour (6-7 heures) à raison de 5 jours par semaine. Quant au cours à temps partiel, c'est souvent la moitié d'heures du temps plein à raison de deux jours par semaine ${ }^{14}$. La durée de formation varie d'un apprenant à l'autre dépendamment de son niveau et du niveau visé.

Tableau 1 - Recueil des données des apprenants sur lesquels nous avons fait l'observation.

\begin{tabular}{|c|c|c|c|c|}
\hline $\begin{array}{l}\text { Type de } \\
\text { cours }\end{array}$ & $\begin{array}{l}\text { Cours de } \\
\text { groupe de } 7\end{array}$ & $\begin{array}{l}\text { Cours } \\
\text { individuel (1) }\end{array}$ & $\begin{array}{l}\text { Cours } \\
\text { individuel (2) }\end{array}$ & duel (3) \\
\hline
\end{tabular}

\footnotetext{
${ }^{11}$ Pour voir le Guide pédagogique du Programme de français langue seconde PFL2, consulter le livre en PDF en ligne: http://publications.gc.ca/collections/collection_2014/efpc-csps/SC102-2-49-2-2006-fra.pdf

${ }^{12}$ Les cahiers des Sessions préparatoires 1 et 2 , Niveau $C$, sont accessibles en ligne en format PDF aux liens suivants: http://publications.gc.ca/collections/collection_2014/efpc-csps/SC102-1-14-1-2007-fra.pdf http://publications.gc.ca/collections/collection_2014/efpc-csps/SC102-1-14-2-2007-fra.pdf

${ }^{13}$ Pour voir un des cahiers de préparations à l'évaluation de l'expression écrite, consultez le lien suivant: http://publications.gc.ca/collections/collection_2018/efpc-csps/SC103-29-4-2016-fra.pdf

${ }^{14}$ Une classe ou un apprenant à temps plein ne peut pas avoir un seul enseignant: il lui en faut deux qui partagent les 7 heures de cours quotidiennes.
} 


\begin{tabular}{|c|c|c|c|c|}
\hline Âge & $\begin{array}{l}\text { Adultes }(+ \text { de } \\
27 \text { ans) }\end{array}$ & $\begin{array}{l}\text { Adulte (dans } \\
\text { la } \\
\text { quarantaine) }\end{array}$ & $\begin{array}{l}\text { Adulte (avant } \\
\text { la retraite }{ }^{15} \text { ) }\end{array}$ & $\begin{array}{l}\text { Adulte (dans } \\
\text { la } \\
\text { cinquantaine) }\end{array}$ \\
\hline Sexe & $\begin{array}{l}4 \text { femmes et } 3 \\
\text { hommes }\end{array}$ & Homme & Femme & Homme \\
\hline Durée & $\begin{array}{l}12 \text { semaines / } \\
7 \mathrm{~h} / \text { jour }\end{array}$ & 12 semaines & 6 semaines & 8 semaines \\
\hline Temps & Plein & Plein & Partiel & Partiel \\
\hline Compétence & Écrit et oral & Écrit et oral & Oral & Oral \\
\hline Niveau visé & $\begin{array}{l}\text { Possèdent B } \\
\text { et préparent le } \\
\mathrm{C}\end{array}$ & $\begin{array}{l}\text { Possède } A \text { et } \\
\text { prépare } B\end{array}$ & $\begin{array}{l}\text { Maintenir le } \\
\text { niveau C }\end{array}$ & $\begin{array}{l}\text { Possède A et } \\
B \text { et prépare } \\
\text { C }\end{array}$ \\
\hline $\begin{array}{l}\text { Langues } \\
\text { acquises }\end{array}$ & $\begin{array}{l}\text { Anglais: } 3 \\
\text { per. } \\
\text { Anglais + } \\
\text { autre langue: } \\
4 \text { pers. }\end{array}$ & Anglais & Anglais & Anglais \\
\hline $\begin{array}{l}\text { Statut } \\
\text { professionnel }\end{array}$ & $\begin{array}{l}\text { Gestionnaires, } \\
\text { chefs de } \\
\text { projets, } \\
\text { Ingénieurs et } \\
\text { comptable }\end{array}$ & $\begin{array}{l}\text { Analyste } \\
\text { économique }\end{array}$ & Directrice & Directeur \\
\hline
\end{tabular}

Il est temps maintenant de donner une idée plus claire de la situation d'enseignement-apprentissage et de la relation enseignant-apprenant. En tant qu'enseignant, il est essentiel de prendre en considération que les apprenants sont des adultes et fonctionnaires du Gouvernement fédéral du Canada. En conséquence, l'apprentissage du français langue seconde a des objectifs spécifiques purement professionnels. L'enseignant doit donc être guidé par quelques principes andragogiques.

Premièrement, les apprenants sont des adultes et la relation apprenant-enseignant ne doit pas être une relation hiérarchisée comme c'est le cas avec les enfants ou adolescents. Nos apprenants adultes doivent être pleinement impliqués dans le processus d'apprentissage. Dès le début des cours, ils doivent exprimer clairement leurs objectifs de formation, leurs points forts qui aident à progresser, leurs difficultés qu'on doit surmonter. Parfois, ils participent même à la planification de leurs cours.

Deuxièmement, il importe de prendre en compte l'expérience de l'apprenant. Tout au long de la période d'apprentissage, l'apprenant fait souvent recours à ses propres expériences. À l'examen final du programme PFL2, qui ne se passe pas avec l'enseignant mais avec des examinateurs de l'École de la fonction publique, l'apprenant doit démontrer ses compétences en présentant des situations réelles de sa vie professionnelle, car c'est autour du sujet de l'emploi que se passe l'examen. En conséquence, les échanges entre l'enseignant et l'apprenant proviennent de la vie réelle de l'apprenant.

\footnotetext{
${ }^{15}$ Certains apprenants à la fin de leur carrière souhaitent maintenir ou obtenir le niveau $\mathrm{C}$ qui lui garantira une bonne retraite.
} 
Troisièmement, le contenu du programme PFL2 est basé sur l'apprentissage par des situations professionnelles concrètes. L'apprenant adulte attend de l'enseignant de le guider vers le concret en lui donnant les occasions de faire les mises en pratique pour améliorer ses compétences. Il a besoin d'être convaincu et doit aussi convaincre pour réussir.

Quatrièmement, l'apprenant adulte a la conscience de la valeur du temps. Dès le début de la formation linguistique, il sait qu'il a des objectifs à atteindre et une durée limitée et précise. Par conséquent, il manifeste systématiquement un intérêt à être attentif, interactif et à partager ses connaissances et expériences. Il est donc censé terminer sa formation à temps et être prêt à passer l'examen. Cependant, cela peut avoir un impact négatif chez certains apprenants; plus l'examen s'approche, plus ils se stressent. L'enseignant doit donc régulièrement encourager l'apprenant et lui remonter le moral.

En somme, l'enseignement aux apprenants adultes est un travail interactif encadré par des principes andragogiques que l'institution linguistique doit souligner à l'enseignant. Et étant donné que l'enseignement aux adultes est un enseignement individualisé, l'enseignant est informé des spécificités de chaque apprenant et doit ne pas seulement maîtriser le programme PFL2 mais également avoir une bonne maîtrise des aspects relationnels; ces apprenants sont considérés pour ces établissements linguistiques comme des «clients », les enseignants sont appelés des formateurs et on parle de la formation au lieu de l'enseignement.

Enfin, cette présentation du contexte de l'étude ainsi que le matériel utilisé et les principes andragogiques permet de mettre en évidence la complexité d'une situation d'apprentissage d'adultes pour des objectifs professionnels et l'influence de l'anglais comme première langue officielle sur l'apprentissage du français comme langue seconde.

\section{INTERFÉRENCE LEXICO-SÉMANTIQUE}

Parmi les domaines d'interférence, le lexique représente un champ d'interférence important qui influence souvent l'aspect sémantique de la langue seconde. La définition de la sémantique et la détermination de son champ d'étude sont encore plus controversées que celles de l'étude du transfert. La grande majorité des travaux sur le transfert sémantique publiés par des chercheurs comme Ellis (1995), Henriksen (1999), Hulstijn, Hollander et Greidanus (1996), Pica (2002), Jiang (2002 ; 2004), portent essentiellement sur le processus psycho-cognitif. À titre d'exemple, pour Jiang (2004), l'apprenant adulte peut s'appuyer sur son système sémantique préétabli. D'autres chercheurs s'intéressent au rôle de la linguistique dans le processus du transfert. Ijaz (1985), par exemple, étudie des groupes d'apprenants anglophones et d'apprenants d'anglais et examine la façon dont ces deux groupes d'apprenants sont influencés par la langue première quant à la signification qu'ils assignent à certains mots. L'interférence sémantique se présente donc quand la connaissance de la langue première influence négativement la production de la langue seconde, c'est-à-dire, lorsqu'elle pose une confusion de sens. Nous avons relevé un grand nombre d'interférences lexico-sémantiques qui posaient un problème de compréhension de l'énoncé. Le tableau 2 contient quelques exemples de ces interférences lexico-sémantiques.

Tableau 2 - Quelques interférences lexico-sémantiques. 


\begin{tabular}{|c|c|c|c|c|}
\hline $\begin{array}{ll}\text { Le mot ou } \\
\text { l'expression en } \\
\text { français }\end{array}$ & $\begin{array}{l}\text { Exemple } \\
\text { d'interférence }\end{array}$ & La correction & $\begin{array}{ll}\text { Le mot } & \text { ou } \\
\text { expression } & \text { en } \\
\text { anglais } & \\
\end{array}$ & $\begin{array}{l}\text { L'équivalent en } \\
\text { français }\end{array}$ \\
\hline 1. Montant & $\begin{array}{l}\text { Au bureau, on } \\
\text { gaspille un } \\
\text { grand montant } \\
\text { de papiers. }\end{array}$ & $\begin{array}{l}\text { Au bureau, on } \\
\text { gaspille une } \\
\text { grande quantité } \\
\text { de papiers. }\end{array}$ & Amount & Quantité \\
\hline 2. Position & $\begin{array}{l}\text { J'ai obtenu ma } \\
\text { position en } \\
2014 \text {. }\end{array}$ & $\begin{array}{l}\text { J'ai obtenu } \\
\text { mon poste en } \\
2014 \text {. }\end{array}$ & Position & Poste \\
\hline 3. Librairie & $\begin{array}{l}\text { Dans notre } \\
\text { ministère, nous } \\
\text { avons une } \\
\text { petite librairie. }\end{array}$ & $\begin{array}{l}\text { Dans notre } \\
\text { ministère, nous } \\
\text { avons une } \\
\text { petite } \\
\text { bibliothèque. } \\
\end{array}$ & Library & Bibliothèque \\
\hline 3. Le degré & $\begin{array}{l}\text { J'ai le degré de } \\
\text { maîtrise en } \\
\text { économie. }\end{array}$ & $\begin{array}{l}\text { J'ai le diplôme } \\
\text { de maîtrise en } \\
\text { économie. }\end{array}$ & Degree & Diplôme \\
\hline $\begin{array}{l}5 . \\
\text { fiscale }\end{array}$ & $\begin{array}{l}\text { À la fin de } \\
\text { l'année fiscale, } \\
\text { je dois préparer } \\
\text { un rapport. }\end{array}$ & $\begin{array}{l}\text { À la fin de } \\
\text { l'année } \\
\text { financière, je } \\
\text { dois préparer } \\
\text { un rapport. }\end{array}$ & Fiscal year & $\begin{array}{l}\text { Année } \\
\text { financière }\end{array}$ \\
\hline 7. Agressif & $\begin{array}{l}\text { Ma } \\
\text { gestionnaire est } \\
\text { agressive. }\end{array}$ & $\begin{array}{l}\text { Ma } \\
\text { gestionnaire est } \\
\text { dynamique. }\end{array}$ & Agressive & $\begin{array}{l}\text { Énergique/ } \\
\text { dynamique }\end{array}$ \\
\hline 8. Sauver & $\begin{array}{lr}\text { Je dois } & \text { sauver } \\
\text { de } & \text { l'argent } \\
\text { pour } & \text { les } \\
\text { vacances. }\end{array}$ & $\begin{array}{lr}\text { Je } & \text { dois } \\
\text { épargner } & \text { de } \\
\text { l'argent } & \text { pour } \\
\text { les vacances. }\end{array}$ & Save & Épargner \\
\hline $\begin{array}{l}\text { 9. manquer } \\
\text { quelqu'un }\end{array}$ & $\begin{array}{l}\text { Je te manque } \\
\text { beaucoup. }\end{array}$ & $\begin{array}{l}\mathrm{Tu} \text { me } \\
\text { manques } \\
\text { beaucoup. }\end{array}$ & $\begin{array}{l}\text { I miss you a } \\
\text { lot. }\end{array}$ & $\begin{array}{l}\mathrm{Tu} \text { me } \\
\text { manques } \\
\text { beaucoup. }\end{array}$ \\
\hline $\begin{array}{l}10 . \\
\text { Actuellement }\end{array}$ & $\begin{array}{l}\text { Actuellement, } \\
\text { j'aime les } \\
\text { défis. }\end{array}$ & $\begin{array}{l}\text { En fait, j'aime } \\
\text { les défis. }\end{array}$ & $\begin{array}{l}\text { Actually, I love } \\
\text { challenges. }\end{array}$ & En fait \\
\hline
\end{tabular}

Nous constatons dans le tableau 2 que plusieurs cas d'interférence lexicale influencent l'aspect sémantique de l'énoncé, comme les exemples de 1 à 8. Dans le dernier cas de ce tableau, il s'agit de l'ordre des éléments constitutifs de la séquence française. "Je te manque beaucoup » pour " Tu me manques beaucoup ». La première personne du singulier «Je » se substitue à la deuxième personne du singulier « Tu » et cette substitution entraîne un changement au niveau de conjugaison du verbe et un changement sémantique. Ce transfert négatif vient certainement de l'interférence de l'anglais «I miss you». Les apprenants qui commettent ce type d'interférence 
connaissent le verbe "manquer » utilisé pour exprimer ce sentiment envers quelqu'un mais ils ne savent pas comment l'utiliser, alors le transfert négatif de l'anglais «I miss you » est présent. Pour Nagy et Scott (2004), la connaissance lexicale n'est pas limitée à la dimension déclarative mais c'est aussi un savoir procédural. Il ne suffit pas seulement de connaître les mots mais de les utiliser de façon correcte pour que l'énoncé ait un sens.

Word knowledge is applied knowledge. A person who knows a word can recognize it, and use it, in novel contexts, and uses knowledge of the word, in combination with other types of knowledge, to construct meaning for a text ${ }^{16}$.

Certaines interférences sémantiques sont produites du fait qu'un mot dans la langue première a deux équivalences dans la langue seconde. À l'intérieur du système linguistique français, il est plus facile pour un apprenant de mélanger entre par exemple « savoir » et «connaître » alors que dans sa langue première il n'y a que le mot to know. Nous avons relevé ce problème fréquemment chez les apprenants débutants et moins fréquemment chez les niveaux intermédiaires et avancés. L'analogie entre des mots en français et anglais peut aussi causer des interférences. Prenons l'exemple $n^{\circ} 3$ dans le tableau 2 que nous avons relevé pendant le cours individuel $(1)^{17}$ : les mots librairie et library. Si on suit l'analyse sémantique dans le tableau 3 ci-dessous, on peut bien comprendre les raisons des interférences.

Tableau 3 - Des exemples d'analogie des noms anglais et français qui causent des interférences.

\begin{tabular}{|l|l|l|}
\hline Description de l'objet & Nom en français & Nom en anglais \\
\hline $\begin{array}{l}\text { Boutique où on vend des } \\
\text { livres }\end{array}$ & Librairie & Bookstore \\
\hline Meuble pour ranger & Bibliothèque & Bookcase \\
\hline $\begin{array}{l}\text { Bâtiment où on conserve } \\
\text { des livres }\end{array}$ & Bibliothèque & Library \\
\hline
\end{tabular}

Ce que nous pouvons constater dans le tableau 3, c'est que les apprenants ne font pas de distinction entre bibliothèque et librairie et utilisent librairie dans les deux sens en raison d'un côté la similitude entre librairie et library et d'un autre côté sa simplicité morphologique par rapport à bibliothèque. Selon Meara (2005) ${ }^{18}$, 1'apprenant de la langue seconde n'a pas l'aisance des locuteurs natifs avec certains mots, notamment ceux pour lesquels il n'y a pas d'équivalence dans la langue première. Il va donc éviter de les utiliser et va préférer les mots plus fréquents et ceux qui sont morphologiquement plus simples (Meara, 1984) ${ }^{19}$.

\footnotetext{
${ }^{16}$ Nagy, W. E., et Scott, J. A. (2000), Vocabulary processes, In M. L. Kamil, P. Mosenthal, P. D. Pearson, $\&$ R. Barr (Eds.), Handbook of reading research (Vol. 3, pp. 269-284), p. 273, Mahwah, NJ: Erlbaum.

${ }^{17}$ Voir le tableau 1.

${ }^{18}$ Meara, Paul, «Lexical frequency profile: A Monte Carlo analysis », Applied Linguistics, vol. 26 (1), p. 32-47, Oxford University Press, 2005.

${ }^{19}$ Meara, Paul, « The Study of Lexis in Interlanguage », In A. Davis, C. Criper et A. P. R. Howatt (éd.), p. 225-235. Edinburgh: Edinburgh University Press, 1984.
} 
Le problème d'interférence sémantique se manifeste aussi lorsque certains mots qui proviennent de la même origine gréco-latine, mais leurs développements prennent des directions différentes et des emplois différents. Par exemple, les apprenants ont tendance à utiliser souvent le mot actuellement à la place de en fait ou vraiment etc. : (Actuellement, j'aime les défis) au lieu de (En fait, j'aime les défis) car en fait est un des sens du mot anglais actually. Cet exemple d'interférence que nous avons relevé chez un apprenant dont la langue maternelle est l'anglais ${ }^{20}$ illustre également le problème de similitude entre l'anglais et le français. Ce type d'erreur est généralement considéré dans le système éducatif canadien comme anglicisme.

L'interférence sémantique que les apprenants d'une langue seconde produisent crée une ambigüité qui menace parfois la clarté de l'énoncé ou du discours, et c'est pourquoi elle est considérée comme la plus dangereuse de toutes les interférences.

\section{L'INTERFÉRENCE PHONÉTIQUE ET PHONOLOGIQUE}

Nous avons considéré comme interférences phonologiques, les erreurs relevant d'une prononciation des finales " à l'anglaise ", les erreurs résultant d'une mauvaise discrimination auditive et les autres erreurs affectant la phonétique du mot mais n'appartenant pas aux catégories précédentes.

Le cas le plus représentatif est le « $\mathrm{s} »$ du pluriel en français. Certains apprenants débutants prononcent le « $\mathrm{s}$ » du pluriel en français comme par exemple [lezetaps] au lieu de [lezetap]), [ãplwajes] au lieu [ãplwaje]. En général, avec le temps et suite aux corrections, on voit progressivement la disparition du « s ». Cependant, quelques cas bien étonnants persistent pour longtemps, voire pour toujours. Nous en avons eu des étudiants qui ont fini leur formation tout en gardant le « $\mathrm{s} »$ du pluriel. C'est le cas de l'apprenant du cours individuel $(1)^{21}$. Il est clair que l'influence de l'anglais sur le français dans cette erreur de prononciation est bien forte chez l'apprenant.

Parlant des erreurs interférentielles dans ce domaine, dans le cas concret de notre recherche, portent essentiellement, d'après notre observation, sur le système vocalique. La cause qui provoque des confusions entre les deux systèmes phonétiques, celui de l'anglais et celui du français, est l'absence des quatre voyelles nasales comme $/ \tilde{\varepsilon} /, / \tilde{e} /, / \tilde{\jmath} /$, $/ \tilde{\mathbf{a}} /$ en anglais. Les apprenants de FLS au niveau avancé font moins d'erreurs phonétiques que les débutants. Ceux-ci éprouvent des difficultés à la prononciation des voyelles et l'accent anglais est souvent présent dans leurs phrases.

\section{L'INTERFÉRENCE SYNTAXIQUE}

Les interférences syntaxiques dans les productions orales sont aussi présentes. Les apprenants utilisent parfois certaines structures de l'anglais dans les phrases en français dans tous les aspects de la syntaxe : ordre des mots, l'usage des déterminants, des pronoms, des adjectifs, les accords, le temps etc. Voici par exemple quelques énoncés produits par ces fonctionnaires apprenants du français : ma préférée tâche (ordre), j'étais né à Ottawa (temps), ma gestionnaire a commencé sa projet (accord), Je lave mes mains (syntaxe -pronominal). Les erreurs de syntaxe sont très fréquentes chez les étudiants anglophones, particulièrement les verbes pronominaux, les prépositions et les pronoms

\footnotetext{
${ }^{20}$ Voir le tableau 1, cours de groupe.

${ }^{21}$ Voir le tableau 1, cours individuel (1).
} 
COD et COI. Les erreurs syntaxiques commises dans l'utilisation des verbes pronominaux et des prépositions sont liées au fait que ceux-ci n'existent pas en anglais. Les pronoms « en » et "y », étant inconnus en anglais, posent un grand problème pour les apprenants anglophones. Ainsi, ils construisent une phrase, par exemple « je pense à ma pension » disant «je lui pense » ou «je pense à lui » à la place de « j’y pense ».

\section{Les prépositions:}

Il convient de signaler qu'au cours de nos recherches sur l'apprentissage du français langue seconde, nous avons pu constater que l'influence de l'anglais est très fréquente dans l'utilisation des prépositions. Essayons d'expliquer pourquoi il en est ainsi. En premier lieu, nous constatons que, là où la structure du français présente des points de faiblesse (irrégularités, manque d'optimalité entre le sens et son expression formelle, etc.), l'anglais, la langue première des fonctionnaires, peut présenter des équivalents plus réguliers.

Abordons donc cet exemple tiré de notre recherche sur l'usage des prépositions. Il s'agit du remplacement de la préposition chez par la préposition de lieu générique à ou par des locutions prépositionnelles telles que à la maison de X, à sa maison, etc. En voici quelques exemples :

1. Je suis allé à mes amis (à la place de chez mes amis). " I went to my friends' house. »

2. Samedi, je vais aller à la maison de Mike (à la place de chez Mike). « I am going to go to Mike's house. »

La préposition chez est sémantiquement une préposition spécialisée, car elle exprime la notion de mouvement vers un domicile ou un professionnel (dentiste, médecin, boucher, coiffeur etc.). Or, la locution prépositionnelle « à la »est une locution prépositionnelle générique, car on peut l'utiliser dans plusieurs situations. Remarquons dans le dernier exemple ci-dessus que l'apprenant l'utilise à la place de la préposition spécialisée.

On peut parler d'un autre cas d'interférence chez les fonctionnaires anglophones contraire à ce premier cas quant à l'utilisation des prépositions. Les apprenants emploient la préposition sur à la place de $a ̀$ pour introduire un complément de lieu, particulièrement en désignant une chaîne de télévision ou une radio. Souvent, nous les entendons construire des phrases comme par exemple j'ai vu l'annonce sur la télévision à la place de à la télévision. L'anglais utilise la préposition on, l'équivalent de sur en français, et l'emploi de sur résulte du transfert indirect de l'usage de on. Ce qui est étonnant dans le cas présent, c'est que ce type d'interférence correspond à un processus de complexification. En effet, l'apprenant choisit une préposition spécialisée sur à la place d'une préposition générique $\grave{a}^{22}$. Ce type d'interférence se manifeste chez tous les apprenants canadiens du tableau 1 , souvent chez ceux du niveau A et B et parfois chez ceux qui préparent ou maintiennent le $\mathrm{C}$.

\section{Le genre}

Étant donné le caractère largement imprévisible du genre en français (le genre du

\footnotetext{
22 Voir Raymond Mougeon, «La question de l'interférence de l'anglais à la lumière de la sociolinguistique ", in Langue, espace, société. Les variétés du français en Amérique du Nord, Sainte-Foy, Québec, Presses de l'Université Laval, 1994, p. 25-40. https:/www.erudit.org/livre/CEFAN/19941/000379co.pdf
} 
signifiant et aussi du signifié) et l'absence du genre du signifiant en anglais, le genre des noms est une source d'erreurs interférentielles chez nos apprenants anglophones. À part les erreurs de genre du masculin et féminin que l'apprenant du français langue seconde commet dans son processus d'apprentissage, nous avons constaté des erreurs purement interférentielles. La méconnaissance du genre en français les pousse à commettre des erreurs d'interférence. Le genre en anglais est presque limité au caractère humain father, mother, girle, boy, wife. Quant aux noms des métiers comme the teacher, the dentist, le genre fait référence au signifié (humain aussi). Dans le genre des adjectifs possessifs, les erreurs interférentielles sont très fréquentes. Elles se manifestent à tous les niveaux, mais elles sont moins fréquentes au niveau avancé. Prenons l'exemple suivant : "Le bureau de ma directrice est ouvert à tous les employés» devient chez une apprenante "sa bureau est ouverte à tous les employés ", parce que directrice est féminin et en anglais cela devient «her office is closed ». Les erreurs dans le choix des adjectifs possessifs viennent donc de l'interférence de leur première langue, car en anglais l'accord se fait avec le possesseur et non pas avec le nom de l'objet possédé. Cependant, nous remarquons une amélioration nette chez les apprenants qui ont une bonne connaissance du système grammatical de l'anglais, car ils font recours à la comparaison grammaticale comme stratégie d'apprentissage.

\section{Les pronoms relatifs}

Dans la phrase complexe et au niveau de la subordination, la transformation relative semble figurer parmi les erreurs les plus tenaces chez nos apprenants anglophones. Les pronoms relatifs constituent une des sources de l'interférence en raison des divergences entre le système des relatifs français et anglais. Certes, le pronom relatif dans les deux systèmes introduit une proposition subordonnée, mais la particularité du français est que la forme des pronoms dépend de leur fonction (qui sujet, que: COD, dont: COI, etc.), alors qu'en anglais, le pronom relatif which, par exemple, peut changer de fonction (sujet et $\mathrm{COD}$ et $\mathrm{COI}$ ). De plus, le français possède deux catégories : pronoms relatifs simples et composés. Ainsi les apprenants fonctionnaires anglophones commettent souvent des erreurs à l'utilisation du pronom relatif simple dont et de tous les pronoms relatifs composés. Bien que dont corresponde dans certains cas d'utilisation (comme complément de nom : Le jeune dont le sac est bleu est mon voisin) à whose en anglais, ce pronom relatif constitue une grande difficulté pour nos apprenants. Nous sommes arrivés à une constatation quasi-commune chez nos apprenants anglophones même ceux qui ont un niveau très avancé en français langue seconde. Ils remplacent fréquemment le pronom dont par le pronom que. Même si parfois, ils arrivent à utiliser dont quand on leur demande de construire des phrases simples, il n'est pas le cas dans un discours pour répondre à des questions d'opinion sur la carrière, les tâches professionnelles, la fonction publique, etc.

Quant aux pronoms relatifs composés (lequel, laquelle, lesquels et lesquelles), les apprenants rencontrent beaucoup de difficultés à les utiliser correctement. Ils ont tendance de les remplacer par qui ou que, et c'est encore plus compliqué quand ils les utilisent avec les prépositions. Nous avons constaté que quand il s'agit d'un de ces pronoms relatifs composés avec la préposition de, les apprenants utilisent parfois dont. Quelques fois, ils démontrent qu'il faut choisir un pronom relatif composé, mais le genre leur pose un autre obstacle. Malgré leur compréhension de la règle des pronoms relatifs, 
toutefois ils prouvent des difficultés à les mettre en pratique dans leur discours spontanément. À l'encontre des pronoms relatifs français, l'anglais utilise les mêmes pronoms relatifs simples (who, whom, which, whose, where, when, that) et y ajoute une préposition (with who, of which, etc.). Dans ces cas, les apprenants commettent des erreurs interférentielles qui trouvent leur origine dans la particularité morphosyntaxique des pronoms relatifs anglais. Ce genre d'interférence se manifeste chez presque tous nos apprenants. Mais au fur et à mesure des préventions et corrections, certains apprenants réussissent à les assimiler, d'autres prouvent des difficultés même au niveau avancé. Chez l'apprenant (2) du tableau 1 par exemple, ces erreurs interférentielles ont résisté pour longtemps bien qu'elle eût déjà le niveau $\mathrm{C}$ et que son objectif était de maintenir son niveau C. Cette difficulté d'assimilation des pronoms relatifs à l'oral est peut-être dû d'un côté au facteur de l'âge de l'apprenant: cette directrice était à 2 ou trois ans de la retraite. D'un autre côté, parce qu'il n'y a pas de changement de la forme de base en anglais, qu'il s'agisse des pronoms relatifs sujet ou complément (pronom relatif qui reprend un humain/ un non humain). La fonction du pronom relatif n'est pas pertinente en anglais dans la mesure où sa morphologie n'en dépend pas. Un même pronom relatif en anglais prend la fonction du sujet et du complément comme par exemple "who/ which/that ». C'est pourquoi on trouve des erreurs interférentielles chez nos apprenants débutants qui utilisent le pronom relatif que comme sujet et complément.

\section{Le verbe pronominal}

Bien que les verbes pronominaux soient rares en anglais, on trouve néanmoins des pronoms réfléchis et des pronoms réciproques comme « herself, himself, each other, etc. » Le verbe pronominal est une des sources de l'interférence que nous avons constaté chez nos apprenants. Nous avons pu dévoiler à travers l'analyse linguistique de notre corpus des phrases relevées pendant nos cours, des erreurs interférentielles en relation avec la forme pronominale des verbes. Parmi ces erreurs interférentielles nous pouvons donner comme exemple «J'ai inscrit» pour «je me suis inscrit ». On voit même parfois une confusion entre le verbe s'inscrire et s'enregistrer. Dans un exercice de grammaire sur le passé composé, le groupe de 7 apprenants dans le tableau 1 devait choisir un verbe parmi inscrire, s'inscrire et s'enregistrer pour compléter la phrase " je ...... à la formation linguistique ». Quatre parmi eux ont choisi le verbe approprié et l'ont bien corrigé. Deux autres ont fait une erreur interférentielle en choisissant le verbe s'enregistrer. Au lieu de dire "je me suis inscrit à la formation linguistique », ils ont répondu " je me suis enregistré à la formation linguistique. " Ces deux apprenants ont en commun l'anglais comme langue maternelle et l'âge : les deux sont à la fin de la cinquantaine. Le septième apprenant à tout simplement commis une erreur grammaticale " $j$ 'ai inscrit à la formation linguistique. " Nous avons également constaté chez nos apprenants canadiens que dans ce type d'exercices, ils peuvent utiliser le verbe pronominal dans des phrases simples et limitées s'ils savent que le verbe en question est pronominal. Mais, s'ils ne savent pas qu'il s'agit d'un verbe pronominal, l'erreur est parfois inévitable. Ils produisent une erreur interférentielle dans une phrase comme « je lave mes mains" qui a source en anglais ( I I wash my hands»). De plus, quand l'apprenant est demandé de faire un discours argumentatif un peu long (de 1 à 2 minutes), l'erreur est fréquente même avec les verbes pronominaux que l'apprenant connait, car dans un discours spontané et long, l'apprenant concentre sur l'information qu'il doit 
transmettre, sur son opinion personnelle et sur la structure du discours, et, par conséquent, commet des erreurs interférentielles dans les verbes pronominaux. C'est ce que nous avons constaté chez les apprenants du niveau avancé comme par exemple au cours individuel (3) du tableau 1.

\section{L'INTERFÉRENCE DES ÉLÉMENTS NON-LINGUISTIQUES}

Il est évident qu'un discours oral comporte, autres que des éléments linguistiques, des éléments non-linguistiques tels que les gestes, les mimiques, les hésitations, les pauses et les changements d'intonation. Il est possible que ces éléments non-linguistiques peuvent révéler des difficultés chez l'apprenant d'une langue seconde pendant la production orale, mais il est parfois difficile pour l'enseignant de préciser s'il s'agit d'une interférence ou non. L'hésitation est un fait inévitable dans le discours oral même dans la langue maternelle. Elle aide à choisir les mots, à organiser le discours et permet de réfléchir aux idées qu'on exprimera. Chez nos apprenants du français langue seconde, les hésitations sont très présentes et sont marquées par des pauses remplies (um, ah etc.), des allongements de voyelles, des répétitions, des ruptures de constructions et des mots d'interférences comme so, wherever, well, you know qu'on entend parfois dans les phrases françaises. Ce qui est étonnant dans les situations d'hésitation, c'est que ces apprenants connaissent souvent l'équivalent de ces mots en français, mais ils utilisent des mots anglais. Cela indique que l'influence de leur langue première est très forte. Voici quelques exemples de phrases que nous avons notées pendant nos cours sur l'hésitation, les pauses et les changements d'intonation :

1. Um j'ai rendu chez moi um je me suis rendu je je suis rendu chez moi /

2. You know, la fonction publique est un grand employeur au Canada et elle investit ah beaucoup dans la formation de ses employés/

3. So, je crois qu'on doit changer um améliorer le processus d'embauchel

Exemples de phrases tirées de notre corpus : le $1^{\text {er }}$ exemple est relevé du cours individuel (1) alors que les deux autres sont relevés du cours de groupe dans le tableau 1.

De plus, on observe que l'intonation de l'anglais de l'Amérique du Nord est souvent très présente dans leurs discours en français, même chez ceux qui ont un niveau avancé en FLS, et il est parfois très difficile de l'effacer. Le transfert de la prosodie de l'anglais au français que fait l'apprenant crée parfois des difficultés à la compréhension de l'énoncé français.

Parfois, certains apprenants utilisent des expressions en français à la manière anglaise pour attirer l'attention de l'interlocuteur comme par exemple vous savez ou tu sais et ils en mettent beaucoup dans un court discours. Ici, il n'y a aucune erreur linguistique et en français on les utilise parfois, cependant quand on les entend se répéter plusieurs fois dans un petit discours d'un apprenant anglophone on peut comprendre que celui-ci ignore qu'en français oral on n'en met pas autant comme en anglais.

Ces marques d'hésitation peuvent révéler d'un côté un effort d'autocorrection ou de reformulation de la part des apprenants et d'un autre côté, elles peuvent révéler une incertitude traduite parfois par des interférences de sa langue première. Néanmoins, les éléments non-linguistiques français sont souvent absents ou utilisés à la manière anglosaxonne. 


\section{L'ASPECT PSYCHOLINGUISTIQUE ET L'INTERFÉRENCE}

Restent à considérer certains facteurs psychologiques qui influencent le transfert de la langue première à la langue seconde chez les apprenants adultes. Il faut d'abord rappeler que les fonctionnaires fédéraux apprennent le français comme langue seconde parce que leur poste ou le poste qu'ils visent exige un certain niveau en langue seconde. Les facteurs psychologiques comme la fatigue, le stress, le statut professionnel, l'oubli, la vieillesse et la durée de formation jouent un rôle important dans leur apprentissage de la langue seconde. Tous ces aspects psycholinguistiques interviennent dans la production des discours dans la langue seconde. Selon les recherches psycholinguistiques, les enfants ont une meilleure perception auditive qui permet une meilleure discrimination, et plus l'apprenant est âgé plus les interférences de la langue première à la langue seconde sont nombreuses. Quand l'apprenant adulte a du stress ou sent de la fatigue, les erreurs et les interférences augmentent et il est difficile de les corriger sur-le-champ. Il est conseillé de changer d'activité et de travailler sur un sujet ou une activité plus amusante. Mais, on a parfois un ou quelques apprenants qui ont souvent du stress pour plusieurs raisons. Dans ce cas, la mission de l'enseignant devient difficile. Il doit travailler en priorité sur le côté psychologique de l'apprenant pour lui remonter le moral et l'aider à mieux se sentir. Le rôle de l'enseignant devient donc très sensible et doit être d'abord psychologique et puis andragogique. L'enseignant doit rassurer l'apprenant, renforcer la confiance en soi et le guider dans le processus d'apprentissage. Certains fonctionnaires commencent leur cours de FLS avec confiance en soi, mais au bout de quelques cours ils se rendent compte que le chemin est un peu plus long qu'ils l'imaginaient. Une autre catégorie d'apprenants vient avec une certaine inquiétude de l'examen. Dès le premier cours, ils commencent à penser à l'examen et aux conséquences s'ils ne réussissent pas. Comme la plupart sont des gestionnaires, ils craignent ne pas réussir et, par conséquent, perdre leur face devant leurs employés et leurs supérieurs. Beaucoup d'entre eux commencent dès les premiers cours à poser des questions à l'enseignant s'il pense qu'ils réussiront l'examen et il est très difficile pour l'enseignant de répondre en se basant sur les premiers cours. Un troisième groupe d'apprenants vient avec une sur-confiance en soi. Ils ont tendance à surévaluer leurs propres connaissances en langue seconde et il est souvent difficile de les convaincre et de les corriger. L'enseignant doit trouver un moyen pour rendre le cours plus plaisant et cela lui demande de ne pas détruire la confiance de l'apprenant et de lui corriger ses erreurs, y compris les interférences.

Dans nos cours du français langue seconde aux adultes et aux enfants ${ }^{23}$, nous avons constaté que nos apprenants adultes ont besoin de plus de temps que les enfants pour assimiler certains aspects linguistiques comme par exemple la prononciation de certaines voyelles. Toutefois, les apprenants adultes ont plus d'aptitudes à comprendre d'autres aspects de la langue seconde comme par exemple les règles grammaticales et la structure syntaxique en générale. Les apprenants adultes ont souvent recours à la comparaison entre la langue seconde et leur langue première pour mieux comprendre la structure de la langue seconde. Ainsi, les apprenants qui connaissent bien ou maîtrisent la structure de leur langue première comprennent la structure de la langue seconde mieux que ceux qui ont moins de connaissances de la structure de leur langue première. Nous sommes arrivés

\footnotetext{
${ }^{23}$ Cette constatation est basée sur notre récente expérience d'enseignement de français à des enfants et adolescents dans quelques écoles en Ontario.
} 
à ce constat après avoir demandé à nos apprenants de faire la comparaison entre la structure grammaticale d'une ou plusieurs phrases de la langue seconde avec celle de leur langue première. Certains donnaient des explications grammaticales pertinentes de leur langue première et arrivaient facilement et rapidement à comprendre le mécanisme de la langue seconde, alors que d'autres, ceux qui connaissent moins les règles grammaticales de leur langue premières, prennent plus de temps pour comprendre et maîtriser les mêmes types de phrases avec moins d'interférences.

Il est à préciser que nous observons les erreurs chez nos apprenants de deux manières : soit l'apprenant ne se rend pas compte de l'erreur, il se voit donc sûr de luimême et commet une erreur, ou bien quand il ne trouve pas le mot adéquat dans le français, il cherche consciemment un moyen pour échapper à cette situation embarrassante et introduit donc un élément de l'anglais. Dans ce dernier cas, on peut voir dans son visage un air d'hésitation ou/et d'incertitude. Nous faisons parfois recours à la linguistique contrastive pour lui expliquer la différence structurale entre les phrases produites en français et l'équivalence en anglais. Notre recours à cette méthode se fait dans certaines situations que nous jugeons nécessaire et nous ne contentons pas de nous focaliser seulement sur la correction des erreurs mais aussi, et surtout, sur la prévention car cela nous semble plus efficace.

\section{ORIGINES DES INTERFÉRENCES}

L'interférence de l'anglais sur le français chez les apprenants canadiens du FL2 peut se manifester à tous les niveaux de production linguistique. La question qui se pose est pourquoi les apprenants canadiens commettent ces interférences? Est-ce que la première langue est la seule raison? Selon Monville-Burston Monique (2013), « la L1 n'est pas la seule source des problèmes dans la progression de l'apprentissage, mais c'est un facteur central. Les apprenants se lancent dans l'aventure de l'acquisition d'une L2 avec comme bagage obligé les schémas solidement enracinés de leur L1 » (Monique MonvilleBurston: 123$)^{24}$. Après cette analyse détaillée ci-dessus et selon nos constats en tant qu'enseignant de français langue seconde, nous pouvons affirmer que l'apparition d'un nombre significatif et varié d'interférences dans notre cas d'étude est attribuée aux raisons suivantes.

Tout d'abord, les similitudes entre les deux langues jouent un rôle majeur. Selon Kocourek (2001: 172), « les deux langues, voisines et cousines, sont fortes dans leurs caractères communs, et riches par la complémentarité de leurs différences ${ }^{25} \gg$. Cela ne veut pas dire que l'analogie entre l'anglais et le français est nuisible à l'apprentissage du FL2, mais les apprenants doivent connaître les ressemblances et les utiliser avec prudence. Les exemples que nous avons recueillis dans nos cours de FL2 illustrent cette difficulté.

Ensuite, l'anglais comme langue maternelle de l'apprenant ou une langue acquise est le deuxième facteur des interférences. Parmi les apprenants canadiens qui ont l'anglais comme première langue officielle, ceux qui ont l'anglais comme langue

\footnotetext{
${ }^{24}$ Monville-Burston Monique, « Complexité et transfert dans l'acquisition du français langue étrangère : le cas des apprenants chypriotes du FLE », Travaux de linguistique, 2013/1 (nº6), p. 97-134. URL: https://www.cairn.info/revue-travaux-de-linguistique-2013-1-page-97.htm

${ }^{25}$ Kocourek, Rostislav. Essais de linguistique française et anglaise : mots et termes, sens et textes, Paris, Édition Peeters. 2001, p. 172.
} 
maternelle font plus d'interférences que ceux qui ont une langue maternelle autre que l'anglais. Car ceux-ci sont des immigrants qui ont acquis l'anglais par l'apprentissage; il est à noter que pour avoir un poste au gouvernement il faut répondre à l'exigence linguistique en présentant une preuve de niveau d'anglais ou de français. Par conséquent, ces apprenants qui ont l'anglais comme langue officielle mais ont une autre langue maternelle possède une expérience d'apprentissage de langues, puisqu'ils avaient appris l'anglais auparavant. Ils sont donc plus conscients à faire la distinction entre les systèmes linguistiques acquis et le système linguistique d'une nouvelle langue en cours d'acquisition.

Puis, comme l'âge de l'apprenant joue un rôle décisif dans l'acquisition d'une nouvelle langue, il l'est aussi dans la production des interférences. Nous parlons ici bien évidement des apprenants adultes seulement. Plus l'apprenant est âgé plus le nombre d'interférences est significatif. L'apprenant plus jeun est plus capable d'assimiler une nouvelle langue, d'anticiper et de corriger ses erreurs interférentielles.

De plus, la connaissance structurelle de l'anglais peut aussi influencer l'apprentissage du français langue seconde. Les apprenants canadiens qui connaissent bien le système grammatical de l'anglais font moins d'interférences en français que ceux qui ne le connaissent pas, car ils font souvent recours à la comparaison entre les deux systèmes linguistiques.

Enfin, le degré d'interférence dépend du stade de l'acquisition de la langue seconde. Les apprenants avancés font naturellement moins d'interférences que les apprenants débutants. Avec plus d'expérience dans l'apprentissage du FL2, le niveau d'interférence chez les apprenants avancés diminue, dans la majorité des cas, de façon considérable.

Après l'analyse des productions des interférences et leurs causes chez les fonctionnaires fédéraux du Canada, il ressort, d'une façon générale, que les paramètres de connaissance de l'anglais ainsi que d'une autre langue, de l'âge, du niveau d'apprentissage du français comme langue seconde et de la durée se sont révélés significatifs. Toutefois, il faut prendre ces interférences comme étant une stratégie d'apprentissage que l'apprenant utilise pour s'exprimer dans la langue seconde.

\section{CONCLUSION}

Bien que cette étude soit centrée sur des fonctionnaires fédéraux apprenant le FLS, sa portée dépasse largement le cadre des employés de la fonction publique du Canada, puisque le français est enseigné comme langue seconde après l'anglais dans presque toutes les régions canadiennes, sauf le Québec où le français est enseigné comme langue première. L'intérêt que nous avons porté à l'étude des erreurs interférentielles chez les apprenants du FLS nous a permis de comprendre que beaucoup de ces erreurs sont dues à l'interférence, connue dans le milieu éducatif au Canada par l'«anglicisme». Il est évident qu'une telle recherche n'est pas facile, mais elle est importante pour aider enseignants et chercheurs à répondre à une question fondamentale de la didactique des langues, à savoir, dans quelle mesure un apprenant adulte peut-il apprendre une langue seconde.

L'apprentissage d'une langue seconde sous tous ses aspects, et pour que l'apprenant ait l'accès à la compétence linguistique et discursive, ne peut évidemment se faire sans interférences. À ce sujet, nous rejoignons totalement Debyser (1970) qui dit 
qu' «Elle (l'interférence) résiste à l'expérience, à l'enseignement, à la pratique, et se prolonge par-delà du perfectionnement de la compétence de (l'apprenant $)^{26}$. » Cependant, à notre avis, il ne faut pas stigmatiser ces types d'erreurs, mais plutôt les accepter en les considérant comme éléments positifs qui aident les apprenants à apprendre une langue seconde. Nous ne prétendons pas ici apporter les solutions clés à ce sujet, mais il est important dans un premier temps de comprendre le «pourquoi » et le «comment» de ces interférences. C'est précisément ce que nous nous sommes attaché de démonter à travers cette étude en analysant les différents types erreurs interférentielles et leurs causes.

Toutefois, l'enseignant ne doit pas s'arrêter à chaque erreur produite par l'apprenant, car cela pourrait freiner le processus d'apprentissage et pour nos apprenants adultes, certains parmi eux se découragent rapidement. Il serait utile de cibler les interférences fréquentes qui entravent le message puis de faire une rétroaction globale. La prévention serait aussi un bon moyen pour diminuer ces erreurs d'interférence et elle pourrait se faire par une discussion préalable avec l'apprenant au début de chaque cours sur la potentialité de ces erreurs. Puisque les interférences ne sont pas fortuites, elles peuvent être analysées, corrigées et même anticipées. C'est dans cette voie qu'il faut travailler pour donner à l'enseignement du français une plus grande efficacité.

Il nous reste à préciser que jusqu'à présent nous avons évoqué les interférences de l'anglais comme langue première sur le français comme langue seconde. Il est possible qu'il puisse y avoir des interférences d'autres langues que l'anglais sur le français surtout que, comme le Canada est un pays d'immigration, certains employés ont une langue maternelle autre que l'anglais, telle que l'espagnol, l'italien, l'arabe, le chinois, le russe, le souahéli, etc. Il serait très intéressant de faire des études contrastives sur cette catégorie d'apprenants à trois volets : la langue maternelle, l'anglais et le français.

\section{BIBLIOGRAPHIE}

Canada. Loi sur les langues officielles, L.C. juillet 1988, ch. 38, partie IV, art. 21, dernière modification le 21 septembre 2017, à jour le 6 juin, 2019, Ministère de la Justice.

Conseil des ministres de l'Éducation (Canada) (2012), Apprentissage et éducation des adultes. Rapport d'étape du Canada en vue du Rapport mondial sur l'apprentissage et l'éducation des adultes (GRALE) de l'UNESCO et de la fin de la Décennie des Nations Unies pour l'alphabétisation (DNUA), Toronto.

Darbelnet, Jean (1976), Le français en contact avec l'anglais en Amérique du Nord, Québec, Presses de l'Université Laval. 207 p.

Bogaards, Paul (2008), On ne parle pas franglais, Bruxelles, De boeck Duculot,

Debyser, Francis (1970), «La linguistique contrastive et les interférences », in Langue française, BELC, Paris, Vol. n 1, pp. 31-61.

Doca, Gheorphe (1981), Analyse psycholinguistique des erreurs faites lors de l'apprentissage d'une langue étrangère, Paris, publication de la Sorbonne.

\footnotetext{
${ }^{26}$ Francis Debyser, «La linguistique contrastive et les interférences », in Langue française, BELC, Paris, 1970, Vol. n 1, pp. 31-61, p. 61.
} 
Kocourek, Rostislav. (2001), Essais de linguistique française et anglaise : mots et termes, sens et textes, Paris, Édition Peeters.

Mackey, William. F. (1976), Bilinguisme et contact des langues, Paris, Edition Klincksieck.

Meara, Paul (2005), «Lexical frequency profile: A Monte Carlo analysis », Applied Linguistics, vol. 26 (1), p. 32-47, Oxford University Press.

Meara, Paul (1984), «The Study of Lexis in Interlanguage », In A. Davis, C. Criper et A. P. R. Howatt (éd.), p. 225-235. Edinburgh: Edinburgh University Press.

Monique, Monville-Burston (2013), «Complexité et transfert dans l'acquisition du français langue étrangère : le cas des apprenants chypriotes du FLE », in Travaux de linguistique, 2013/1 (nº6), p. 97-134.

Mougeon, Raymond (1994), «La question de l'interférence de l'anglais à la lumière de la sociolinguistique », in Langue, espace, société. Les variétés du français en Amérique du Nord, Sainte-Foy, Québec, Presses de l'Université Laval, p. 25-40.

Nagy, W. E., et Scott, J. A. (2000), Vocabulary processes, In M. L. Kamil, P. Mosenthal, P. D. Pearson, \& R. Barr (Eds.), Handbook of reading research (Vol. 3, pp. 269-284), Mahwah, NJ: Erlbaum.

Troubetzkoy, N. (1939), Principes de phonologie, Paris, Klincksieck. 